\title{
Erratum to: The Impact of Chronic Cough: A Cross-Sectional European Survey
}

\author{
Sarah A. F. Chamberlain ${ }^{1,2} \cdot$ Rachel Garrod $^{3} \cdot$ Abdel Douiri $^{4,5} \cdot$ Sarah Masefield $^{6}$. \\ Pippa Powell ${ }^{6}$ Catherine Bücher ${ }^{2,7}$ - Anand Pandyan ${ }^{2,7}$ - Alyn H. Morice ${ }^{8}$. \\ Surinder S. Birring ${ }^{1}$
}

Published online: 12 June 2015

(C) Springer Science+Business Media New York 2015

\section{Erratum to: Lung (2015) 193:401-408 \\ DOI 10.1007/s00408-015-9701-2}

Unfortunately, there is a minor error in Table 1 of the original publication. As for the number of respondents who had 'seen a doctor regarding cough at least once', the corresponding figures should be 1043 (93) instead of 104 (93).

The online version of the original article can be found under doi:10.1007/s00408-015-9701-2.

\section{Surinder S. Birring}

surinder.birring@nhs.net

1 Division of Asthma, Allergy and Lung Biology, King's College London, Denmark Hill Campus, London SE5 9RS, UK

2 School of Health and Rehabilitation, Keele University, Keele, UK

3 King's College London, Denmark Hill Campus, London, UK

4 Department of Primary Care and Public Health Sciences, King's College London, London, UK

5 NIHR Biomedical Centre, King's College London, London, UK

6 European Lung Foundation, Sheffield, UK

7 Institute for Science and Technology in Medicine, Keele University, Keele, UK

8 Hull York Medical School, Castle Hill Hospital, Cottingham, UK 